\title{
Relationship Between Blood Flow Velocity and Blood Suppression Inversion Time for Optimal Visualization of Renal Artery in Spatial Labeling with Multiple Inversion Pulses: In Vitro Study
}

\author{
Yigang Pei ${ }^{1,2}$, Qian $\mathrm{Ye}^{1}$, Juxiong Xiao ${ }^{1}$, Hao Shen ${ }^{3}$, Xueying Long ${ }^{1}$ and Xianjing Peng ${ }^{1,{ }^{*}}$ \\ ${ }^{1}$ Department of Radiology, Xiangya Hospital, Central South University, Changsha, Hunan, P.R. China \\ ${ }^{2}$ Xiangya Hospital, Central South University, Changsha, Hunan, P.R. China \\ ${ }^{3}$ GE Healthcare, Waukesha, WI, USA \\ "Corresponding author: Xianjing Peng, Department of Radiology, Xiangya Hospital, Central South University, P.O. Box: 410008, 87 Xiangya Road, Changsha, P.R. China. Tel: \\ +86-73189753812, Fax: +86-73184327332. Email: pengxianjing@csu.edu.cn
}

Received 2017 December 24; Revised 2018 August 05; Accepted 2018 August 08.

\begin{abstract}
Background: Improving imaging quality of non-contrast enhanced magnetic resonance angiography (NCE-MRA) is essential to those renal function impairment patients especially patients with renal segmental branch disease in the renal parenchyma.

Objectives: To investigate the relation between blood flow velocity (BFV) and blood suppression inversion time (BSP TI) for optimal visualization of the renal artery in NCE-MRA by spatial labeling with multiple inversion pulses (SLEEK) sequence.

Patients and Methods: A catheter (simulated renal artery) was used to perform SLEEK with various BSP TI when injected saline with different velocity (simulated BFV). The relationship between BFV and BSP TI was evaluated by measuring signal-to-noise ratio (SNR) and was confirmed in nine hypertensive patients.

Results: A negative relationship was found in vitro study between BFV and BSP TI with SLEEK sequence $(r=-0.977, \mathrm{P}=0.023)$. For BSP $\mathrm{TI}=200 \mathrm{~ms}$, the simulated renal artery displayed hyper-intensity when simulated BFV $=96-128 \mathrm{~cm} / \mathrm{s}$ and the maximum SNR was found when $B F V=128 \mathrm{~cm} / \mathrm{s}(S N R=0.927)$. For BSP TI $=500 \mathrm{~ms}$, high signal appeared with $B F V=64-128 \mathrm{~cm} / \mathrm{s}$ and the greatest SNR was found with $\mathrm{BFV}=96 \mathrm{~cm} / \mathrm{s}(\mathrm{SNR}=1.732)$. For BSP TI $=800 \mathrm{~ms}$ and $1100 \mathrm{~ms}$, hyper-intensity arose with $\mathrm{BFV}=16-128 \mathrm{~cm} / \mathrm{s}$ and $\mathrm{BFV}=6.4$ $-128 \mathrm{~cm} / \mathrm{s}$ separately, the highest SNR was described when $\mathrm{BFV}=32 \mathrm{~cm} / \mathrm{s}(\mathrm{SNR}=2.256)$ and $\mathrm{BFV}=16 \mathrm{~cm} / \mathrm{s}(\mathrm{SNR}=1.875)$. The different optimal BSP TI with best image quality was discovered in nine hypertensive patients.

Conclusion: An optimal BSP TI based on BFV can improve the ability of delineating renal artery in SLEEK sequence.
\end{abstract}

Keywords: SLEEK, BSP TI, BFV, In Vitro, Renal Artery

\section{Background}

Over the past decade, some non-contrast enhanced MR angiography (NCE-MRA) sequences such as spatial labeling with multiple inversion pulses $(\operatorname{SLEEK})(1,2)$, inflow inversion recovery (IFIR) $(3,4)$, time-spatial labeling inversion pulse (Time-SLIP) $(5,6)$, and steady-state free precession (SSFP) $(7,8)$ have been developed as an alternative to contrast-enhanced MRA (CE-MRA) and computer tomography angiography (CTA) for avoiding radically nephrogenic systemic fibrosis (NSF) or contrast-induced nephropathy (CIN) in renal function impairment patients. They can provide excellent results in the assessment of problematic vessels. Tang et al. (1) found that SLEEK NCE-MRA was better than ultrasound in displaying accessory renal arteries and there was an excellent relation of capacity in depicting transplant renal artery stenosis (TRAS) between SLEEK NCEMRA and digital subtraction angiography (DSA). Lanzman et al. (9) stated that SSFP NCE-MRA was a reliable technique for evaluating TRAS in comparison with DSA. Mohrs et al. (10) found that the specificity and sensitivity of SSFP NCEMRA were $99 \%$ and $75 \%$ in showing renal artery stenoses greater than $50 \%$. However, the image quality of NCE-MRA still needs to improve in the visibility of renal artery, especially for displaying renal segmental branches in the renal parenchyma.

How to improve the NCE-MRA imaging quality for displaying the renal artery has become a hot research topic at present. For this purpose, we have performed SLEEK sequence for displaying the renal artery. In SLEEK, blood suppression inversion time (BSP TI) is defined as the duration from the start time point of the initial inversion pulse to 
the time point that longitudinal magnetization of blood reaches zero (null) point, which was also named as inversion time after the spatial-selective inversion-recovery pulse (ssTI) in Time-SLIP NCE-MRA (11), and TI (Time delay) in SSFP NCE-MRA (10). For distinctly showing the renal artery, Shonai et al. (11) believed that ssTI should be performed with 1200 -1800 ms in Time-SLIP NCE-MRA, and Parienty et al. (8) applied 1100 - 1500 ms in SSFPNCE-MRA. Kurata et al. (12) held a TI with $1800 \mathrm{~ms}$ to visualize the renal artery using time-SLIP. In our previous study, we found that BSP TI which depended on the breathing rate and heart rate should be adopted with $800-1400$ ms to clearly display the renal arteries (13). All the above-mentioned work indirectly suggested that BSP TI may be relative with the blood flow velocity (BFV). However, to the best of our knowledge, it is still unclear whether the applied BSP TI has a correlation with BFV for distinctly delineating renal artery, and even renal segmental branches in the renal parenchyma.

\section{Objectives}

The aim of our study was to disclose the relationship between BSP TI and BFV in SLEEK sequence in vitro, which will be confirmed in nine hypertensive subjects.

\section{Patients and Methods}

\subsection{Simulated Vessel Device}

A round plastic container with a diameter of approximately $15 \mathrm{~cm}$ was taken as human tissue at the kidney level, in which a catheter with an interior diameter of $0.2 \mathrm{~cm}$ was installed as a simulated renal artery (Figure 1) and was imbedded with about $500 \mathrm{~mL}$ pork fat. A terminal of the catheter was connected to a high-pressure syringe (Stellant D, Medrad, Warrendale, PA, USA) for injecting saline with different flow velocity, and the other end was fixed in another $500 \mathrm{~mL}$ plastic container to gather the outflow saline. The catheter was placed along the longitudinal direction paralleled to the scanning bed in our research.

\subsection{Hypertension Patients}

This study was approved by the institutional review board and written informed consent was obtained from all participants prior to examination. Nine hypertensive male patients with similar height $(165-170 \mathrm{~cm})$, weight (60 - 65 $\mathrm{Kg}$ ), and age (40 - 50 years) were selected from 93 consecutive hypertensive subjects diagnosed by a physician (systolic pressure $\geq 140 \mathrm{mmHg}$ and diastolic pressure $\geq 90$ $\mathrm{mmHg}$ ), who were suspected of renal artery stenosis and SLEEK was carried out on them in our prior study (13).

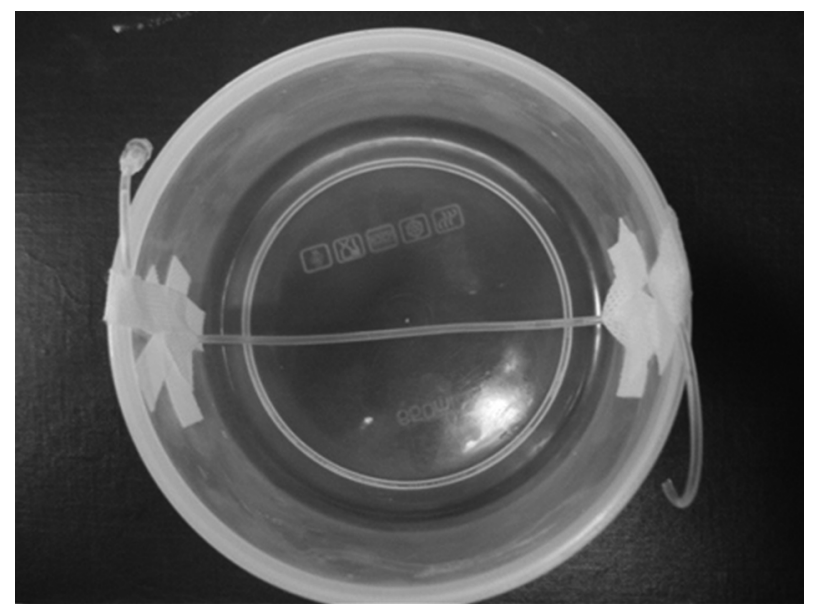

Figure 1. A simulated renal artery (catheter with interior diameter $0.2 \mathrm{~cm}$ ) was installed in a round plastic container (diameter $15 \mathrm{~cm}$ ).

\subsection{MR Angiography}

The vitro studies were performed with a superconductive 1.5T MRI scanner (EXCITE HD, GE Healthcare, Waukesha, WI, USA) using a dual channel phased-array knee coil. In SLEEK NCE-MRA, two orthogonal inversion bands were set to image the simulated vessel based during scanning on the in-flow effect. One $20 \mathrm{~cm}$ width vertical broad inversion band covered the whole container to invert all signals within the coil region to -Mz longitudinal magnetization. The other $10 \mathrm{~cm}$ width transversal inversion band was located on the end of the catheter connected with highpressure syringe to bring the in-flow artery blood back to $+\mathrm{Mz}$ direction $(2,13)$. The diagrams of the SLEEK sequence and the signal acquisition are described in Figure 2.

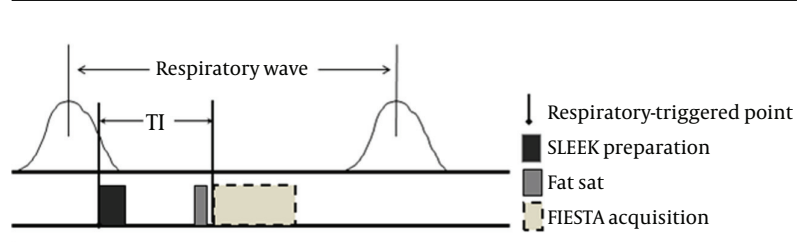

Figure 2. The diagrams of respiratory-triggered spatial labeling with multiple inversion pulses (SLEEK) pulse and signal acquisition with fat saturation 3D fast imaging employing steady-state (FS-3D-FIESTA) sequence.

Saline was injected into the catheter (simulated renal artery) by a high-pressure syringe with flow rates $=0.1,0.2$, $0.5,1.0,2.0,3.0$, and $4.0 \mathrm{~mL} / \mathrm{s}$. The corresponding simulated BFV was 3.2, 6.4, 16, 32, 64, 96, and $128 \mathrm{~cm} / \mathrm{s}$, respectively according to the formula: Simulated $\mathrm{BFV}(\mathrm{cm} / \mathrm{s})=$ flow rate $(\mathrm{mL} / \mathrm{s}) / \pi \mathrm{r}^{2}$. Here $\pi \mathrm{r}^{2}$ was the cross-sectional area of the catheter, and $\mathrm{r}$ was the catheter radius. $1 \mathrm{~mL}=1 \mathrm{~cm}^{3}, \pi=3.14$ 
and $\mathrm{r}=0.1 \mathrm{~cm}$ in our study. Four BSP TIs $(200 \mathrm{~ms}, 500 \mathrm{~ms}$, $800 \mathrm{~ms}, 1100 \mathrm{~ms}$ ) were taken in our study. For each BSP TI, the SLEEK NCE-MRA was performed for the simulated renal arteries with different BFV. The specific image parameters included: Repetition time $(\mathrm{TR})=2.0 \mathrm{~ms}$, echo time $(\mathrm{TE})=$ $1.1 \mathrm{~ms}$, Flip angle $=75$, slice thickness $=0.8 \mathrm{~mm}$, number of slices $=20$, matrix $=128 \times 128$, field of view $(F O V)=20 \mathrm{~cm} \times$ $16 \mathrm{~cm}$, number of excitations $(\mathrm{NEX})=1$, receiver bandwidth $= \pm 31.25 \mathrm{kHz}$. In addition, a false respiratory gating was triggered in whole SLEEK scanning. A SLEEK NCE-MRA with one BSP TI took approximately 40 - 50 seconds.

For hypertensive patients, SLEEK NCE-MRA with three various BSPTIs ( $800 \mathrm{~ms}, 1100 \mathrm{~ms}$ and $1400 \mathrm{~ms}$ ) were carried out to display the renal artery. The scan parameters were identically as follows: $\mathrm{TR}=3.9 \mathrm{~ms}, \mathrm{TE}=2.0 \mathrm{~ms}$, slice thickness $=2 \mathrm{~mm}$, matrix $=224 \times 256, \mathrm{FOV}=38 \mathrm{~cm} \times 30 \mathrm{~cm}$, Flip angle $=75, \mathrm{NEX}=0.80$, sense factor $=2$, receiver bandwidth $= \pm 125 \mathrm{kHz}$, respiratory interval $=1$. A SLEEK NCE-MRA with one BSP TI was took about 3 - 4 minutes, the total SLEEK MR data acquisition time was approximately 10 -12 minutes for each subject.

\subsection{Image Analysis}

The post-processing of all data of SLEEK including simulated vessel and renal artery in hypertensive patients were performed in an imaging workstation (Advantage Workstation 4.4, GE Healthcare, Buc, France). The main post-processing methods were multiple plane reconstruction and maximum intensity projection with the same thickness. The SLEEK images were assessed blindly and randomly.

For each simulated vessel, a region-of-interest (ROI: 8 $12 \mathrm{~mm}^{2}$ ) was placed manually on the most hyper-intense point of the simulated renal artery to obtain signal intensity (SI), and the same ROI size was placed on the nearby background to gain standard deviation (SD) by two experienced radiologists in consensus. The ROIs were drawn three times in different days in a 1-week period and the mean SI and the mean SD were calculated. The signal-tonoise ratio (SNR) was calculated with the formula: SNR = SI/SD. For each BSP TI, the greatest SNR of catheter was decided in various BFVs with SLEEK scan.

For each hypertensive patient, the best image quality was determined by two experienced radiologists in consensus according to the following factors, including best renal artery's SNR, homogeneous vessel signal intensity, sharp and complete delineation of vessel borders, and showing clearly segmental branches in the renal parenchyma. The optimal BSP TI was defined as BSP TI corresponding to the best image quality of the renal artery.

\subsection{Statistical Analysis}

The relationship between BSP TI and BFV was analyzed based on the best SNR by using Pearson's correlation analysis. Statistical analysis was performed using commercially available software (SPSS for Windows, version 13.0; SPSS, Chicago, IL). $\mathrm{P}<0.05$ was considered to indicate a significant difference.

\section{Results}

SLEEK NCE-MRA with various BSP TI was successfully performed and gained different SNR in the simulated renal artery with various BFVs. For SLEEK images with BSP $\mathrm{TI}=200 \mathrm{~ms}$, the simulated vessels appeared hyper-intense when BFVs were $96 \mathrm{~cm} / \mathrm{s}$ and $128 \mathrm{~cm} / \mathrm{s}$, and the corresponding SNRs were 0.852 and 0.927 , respectively. However, they presented hypo-intensity and SNRs were 0.476, 0.447, $0.616,0.238$, and 0.126 , respectively when BFVs were 3.2 $\mathrm{cm} / \mathrm{s}, 6.4 \mathrm{~cm} / \mathrm{s}, 16 \mathrm{~cm} / \mathrm{s}, 32 \mathrm{~cm} / \mathrm{s}$, and $64 \mathrm{~cm} / \mathrm{s}$. For BSP TI = $500 \mathrm{~ms}$, the simulated vessels displayed high signal when BFVs were $64 \mathrm{~cm} / \mathrm{s}, 96 \mathrm{~cm} / \mathrm{s}$, and $128 \mathrm{~cm} / \mathrm{s}$, the corresponding SNRs were $1.119,1.732$, and 1.633 , respectively. In addition, the catheters presented low signal and SNRs were $0.296,0.388,0.232$, and 0.725 , respectively when BFVs were from $3.2 \mathrm{~cm} / \mathrm{s}$ to $32 \mathrm{~cm} / \mathrm{s}$. For BSP TI $=800 \mathrm{~ms}$, catheters showed hyper-intensity if BFVs were from $16 \mathrm{~cm} / \mathrm{s}$ to 128 $\mathrm{cm} / \mathrm{s}$ and the corresponding SNRs were 0.515, 2.256, 1.013, 0.592 , and 0.442 . On the other hand, catheters displayed hypo-intensity and SNRs were 0.178 and 0.327 , respectively when BFVs were $3.2 \mathrm{~cm} / \mathrm{s}$ and $6.4 \mathrm{~cm} / \mathrm{s}$. For SLEEK with BSP $\mathrm{TI}=1100 \mathrm{~ms}$, the simulated vessels presented high signal when BFVs were between $6.4 \mathrm{~cm} / \mathrm{s}$ and $128 \mathrm{~cm} / \mathrm{s}$, the corresponding SNRs were $0.680,1.875,1.577,1.274,0.579$, and 0.225 , respectively. However, the only hypo-intensity presented and SNR was 0.401 when BFV was $3.2 \mathrm{~cm} / \mathrm{s}$ (Figures 3 and $4 \mathrm{~A}$ ) (Table 1). A negative correlation between BFV and BSP TI was found in the in vitro study with SLEEK sequence $(r=-0.977, P=0.023$ ) (Figure 4B).

In nine hypertensive patients, all renal arteries could be displayed when BSP TI was $800 \mathrm{~ms}, 1100 \mathrm{~ms}$, and $1400 \mathrm{~ms}$ with different SNRs, homogeneity, sharpness of vessel borders, and ability of delineating segmental branches in the renal parenchyma. An optimal BSP TI was found for each patient (optimal BSP TI was $800 \mathrm{~ms}$ in two, $1100 \mathrm{~ms}$ in four, and $1400 \mathrm{~ms}$ in three patients) (Figure 5) (Table 2).

\section{Discussion}

With development of MR technology and software, NCE-MRA has gradually obtained an excellent appearance 


\section{Simulated BFV}

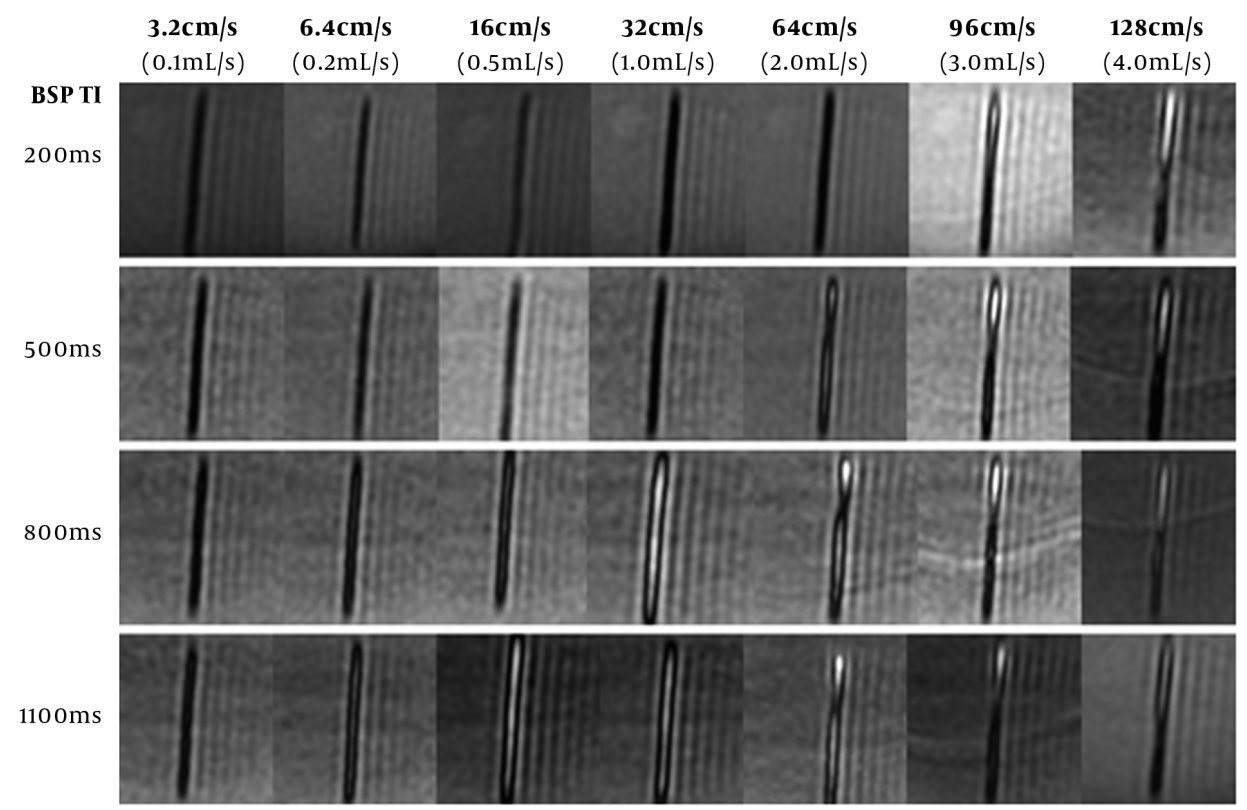

Figure 3. The appearance of the imitated renal artery with different blood flow velocity (BFV) by spatial labeling with multiple inversion pulses (SLEEK) with various blood suppression inversion time (BSP TI). The maximum signal to noise ratio (SNR) was found when $\mathrm{BFV}=128 \mathrm{~cm} / \mathrm{s}$ for BSP TI $=200 \mathrm{~ms}, \mathrm{BFV}=96 \mathrm{~cm} / \mathrm{s}$ for BSP TI $=500 \mathrm{~ms}$, BFV $=32$ $\mathrm{cm} / \mathrm{s}$ for $\mathrm{BSP} \mathrm{TI}=800 \mathrm{~ms}$, and $\mathrm{BFV}=16 \mathrm{~cm} / \mathrm{s}$ for $\mathrm{BSP} \mathrm{TI}=1100 \mathrm{~ms}$. The $\mathrm{BFV}$ had a negative relation with BSP TI was implied.

A

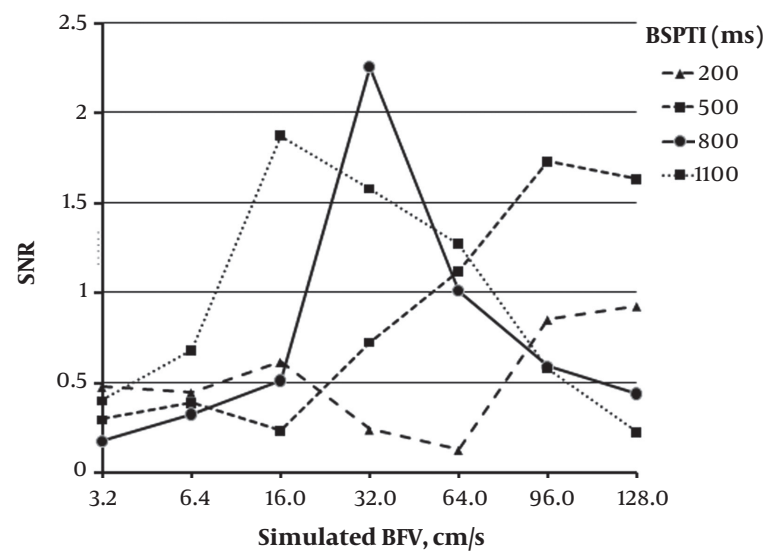

B

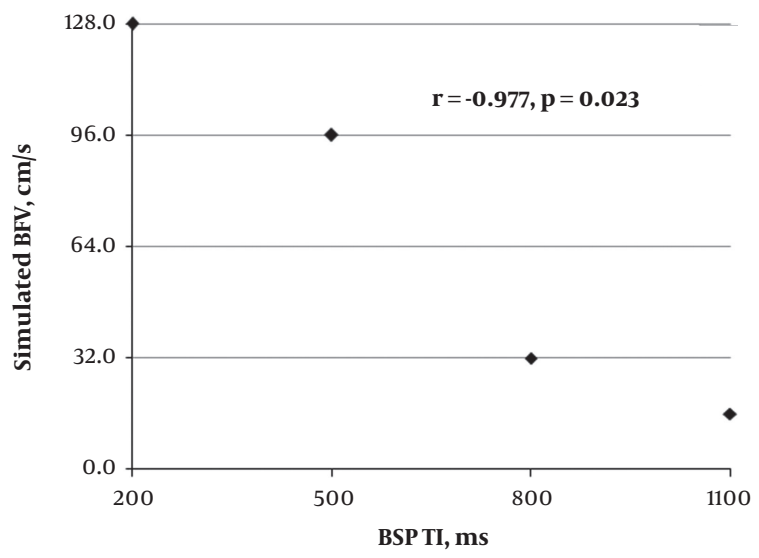

Figure 4. A, The signal to noise ratio (SNR) of the simulated renal artery with different blood flow velocities (BFV) by spatial labeling with multiple inversion pulses (SLEEK) with various blood suppression inversion times (BSP TI); B, Finding of the relationship between BSP TI and BFV. In A, the greatest SNR was 0.927 when $\mathrm{BFV}=128 \mathrm{~cm} / \mathrm{s}$ for BSP TI = $200 \mathrm{~ms}, 1.732$ when $\mathrm{BFV}=96 \mathrm{~cm} / \mathrm{s}$ for BSP TI $=500 \mathrm{~ms}, 2.256$ when $\mathrm{BFV}=32 \mathrm{~cm} / \mathrm{s}$ for BSP TI $=800 \mathrm{~ms}$, and 1.875 when BFV $=16 \mathrm{~cm} / \mathrm{s}$ for BSP TI $=1100 \mathrm{~ms}$. In B, based on the highest SNR for each BSPTI, a negative correction between BFV and BSP TI was found in SLEEK sequence $(r=-0.977, P=0.023)$.

for delineating large blood vessels, including intracranial arteries (14), thoracic aorta (15), renal artery $(1,2)$, and hepatic vessels $(3,16)$. In our study, the renal arteries were presented in coronal plane with SLEEK NCE-MRA sequence, which was a respiratory-triggered 3D fat saturation-fast imaging employing steady-state acquisition (FS-FIESTA) prepared with multiple spatial selective inversion recovery pulses. It is essential to boost the NCE-MRA image quality for those renal artery disease patients. Pei et al. (13) found that the heart and breathing rate can affect the presenting 


\begin{tabular}{|c|c|c|c|c|c|c|c|}
\hline \multirow{2}{*}{ BSP TI } & \multicolumn{7}{|c|}{ Simulated BFV } \\
\hline & $3.2 \mathrm{~cm} / \mathrm{s}$ & $6.4 \mathrm{~cm} / \mathrm{s}$ & $16 \mathrm{~cm} / \mathrm{s}$ & $32 \mathrm{~cm} / \mathrm{s}$ & $64 \mathrm{~cm} / \mathrm{s}$ & $96 \mathrm{~cm} / \mathrm{s}$ & $128 \mathrm{~cm} / \mathrm{s}$ \\
\hline $200 \mathrm{~ms}$ & 0.476 & 0.447 & 0.616 & 0.238 & 0.126 & 0.852 & 0.927 \\
\hline $500 \mathrm{~ms}$ & 0.296 & 0.388 & 0.232 & 0.725 & 1.119 & 1.732 & 1.633 \\
\hline $800 \mathrm{~ms}$ & 0.178 & 0.327 & 0.515 & 2.256 & 1.013 & 0.592 & 0.442 \\
\hline $1100 \mathrm{~ms}$ & 0.401 & 0.680 & 1. 875 & 1. 577 & 1.274 & 0.579 & 0.225 \\
\hline
\end{tabular}

Abbreviations: BFV, blood flow velocity; BSP TI, blood suppression inversion time; SLEEK, spatial labeling with multiple inversion pulses; SNR, signal-to-noise ratio

${ }^{a}$ SNR was the mean value of the greatest SNR for three measurements in different time points. Simulated BFV was calculated with the formula: The simulated BFV (cm/s)

= flow rate $(\mathrm{mL} / \mathrm{s}) / \pi \mathrm{r}^{2}, 3.2 \mathrm{~cm} / \mathrm{s}=0.1 \mathrm{~mL} / \mathrm{s} ; 6.4 \mathrm{~cm} / \mathrm{s}=0.2 \mathrm{~mL} / \mathrm{s} ; 16 \mathrm{~cm} / \mathrm{s}=0.5 \mathrm{~mL} / \mathrm{s} ; 32 \mathrm{~cm} / \mathrm{s}=1.0 \mathrm{~mL} / \mathrm{s} ; 64 \mathrm{~cm} / \mathrm{s}=2.0 \mathrm{~mL} / \mathrm{s} ; 96 \mathrm{~cm} / \mathrm{s}=3.0 \mathrm{~mL} / \mathrm{s} ; 128 \mathrm{~cm} / \mathrm{s}=4.0 \mathrm{~mL} / \mathrm{s}$.

BSP TI: $\quad 800 \mathrm{~ms}$

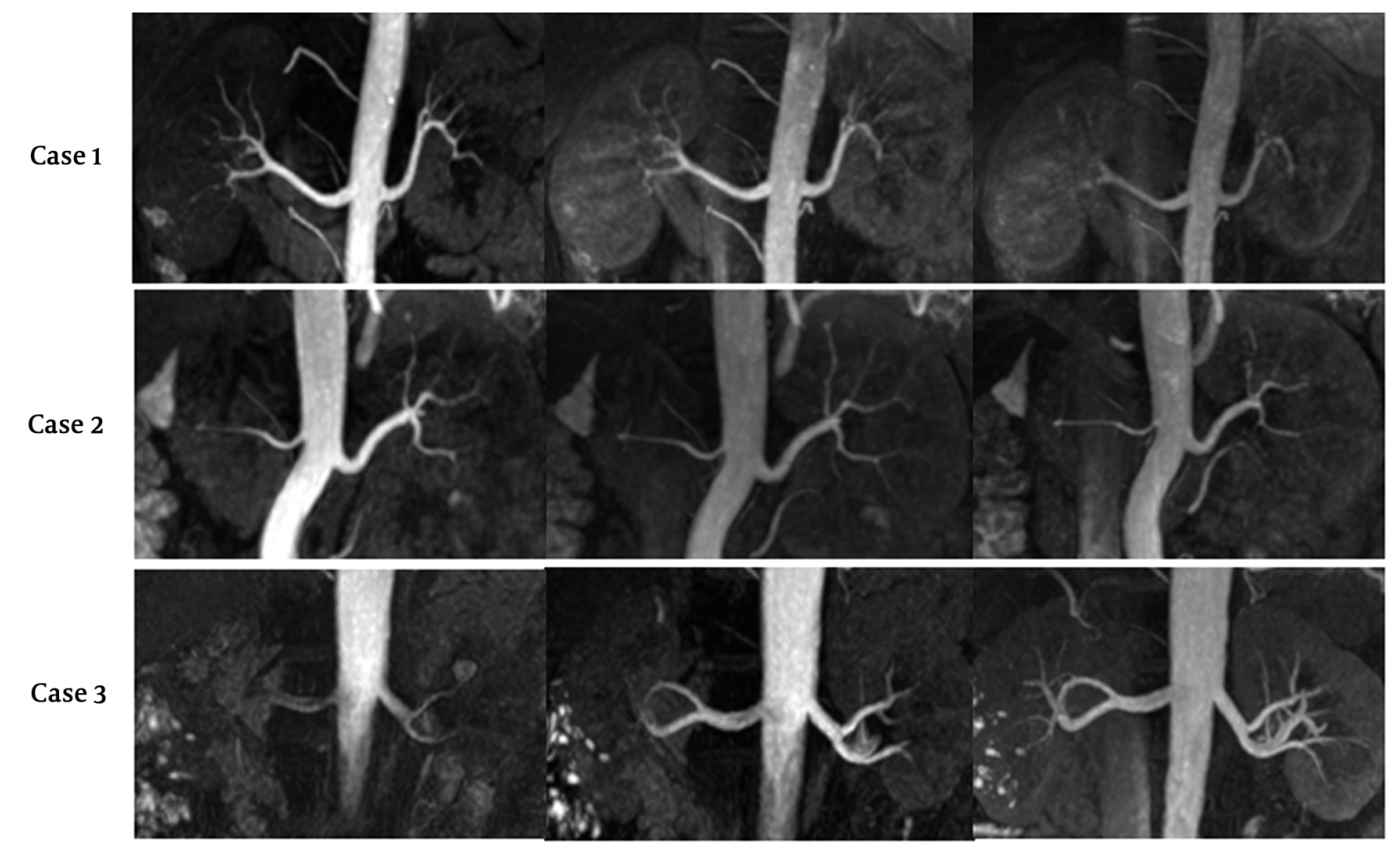

$1100 \mathrm{~ms}$
$1400 \mathrm{~ms}$

Figure 5. The optimal blood suppression inversion time (BSP TI) for best image quality of renal artery in spatial labeling with multiple inversion pulses (SLEEK) sequence in hypertension patients. In three hypertension male patients with similar height, weight and age, the best image quality was found when BSP TI was 800 ms in the first, 1100 $\mathrm{ms}$ in the second, and $1400 \mathrm{~ms}$ for the third patient, which suggested the optimal BSP TI was vital to improve the ability of presenting renal artery in SLEEK sequence.

ability of the renal artery. Kurata et al. (12) deemed that patient age impacted optimal TI for the visualization of renal arteries. All the above pointed out that BSP TI may be related with the BFV.

In our results, a catheter was used to simulate the renal artery and carry out SLEEK with different BSP TIs and BFVs. To our knowledge, this is the first study that has evaluated the relationship between BFV and BSP TI in SLEEK in vitro. Meaningful results were found. First, the catheters appeared low signal when simulated BFV was $3.2 \mathrm{~cm} / \mathrm{s}$. The results illustrated that in SLEEK scan with any BSP TI, the simulated vessel presented hypo-intensity when BFV was so low that the simulated inflow blood could not arrive at the target vessel level. Second, for a given BSP TI, the simulated vessel with various BFVs can show hyper-intensity with SLEEK NCE-MRA. For example, the catheters presented high signal from BFV $=6.4 \mathrm{~cm} / \mathrm{s}$ to $128 \mathrm{~cm} / \mathrm{s}$ for BSP TI $=1100$ ms. It pointed out that the simulated renal arteries with 


\begin{tabular}{|c|c|c|c|c|}
\hline No. & $\operatorname{Age}(y)$ & Height (cm) & Weight (Kg) & The optimal BSP TI (ms) \\
\hline 1 & 42 & 167 & 63 & 1400 \\
\hline 2 & 49 & 166 & 61 & 1100 \\
\hline 3 & 46 & 168 & 60 & 1100 \\
\hline 4 & 40 & 166 & 65 & 800 \\
\hline 5 & 44 & 168 & 63 & 1400 \\
\hline 6 & 47 & 170 & 62 & 1400 \\
\hline 7 & 41 & 170 & 60 & 800 \\
\hline 8 & 47 & 167 & 63 & 1100 \\
\hline 9 & 42 & 165 & 62 & 1100 \\
\hline
\end{tabular}

Abbrevations: BSP TI, blood suppression inversion time; SLEEK, spatial labeling with multiple inversion pulses; SNR, signal-to-noise ratio.

${ }^{\mathrm{a}}$ The optimal BSP TI (ms) was defined as the BSP TI with best renal artery image quality, which included best renal artery SNR, homogeneous vessel signal intensity, sharp and complete delineation of vessel borders, and showing clearly segmental branches in renal parenchyma.

different BFVs could be shown by SLEEK in various SNRs, which can affect the homogeneity of vessel signal intensity and sharpness of renal artery borders. Third, the highest SNR was found when BFV was $128 \mathrm{~cm} / \mathrm{s}$ for BSP TI $=200 \mathrm{~ms}$, $\mathrm{BFV}$ was $96 \mathrm{~cm} / \mathrm{s}$ for BSP TI $=500 \mathrm{~ms}$, BFV was $32 \mathrm{~cm} / \mathrm{s}$ for BSP $\mathrm{TI}=800 \mathrm{~ms}$, and $16 \mathrm{~cm} / \mathrm{s}$ for $1100 \mathrm{~ms}$. It suggested that the $\mathrm{BFV}$ has a negative relationship with BSP TI. The reason was that a fast BFV means shortened time of inflow blood from the heart to the renal artery level, which needs a short echo time to match for gaining a high signal in the renal artery. That means the optimal BSP TI must increase for obtaining the best SNR when BFV decreases. So, adopting an optimal BSP TI can improve the ability of clearly displaying the renal artery.

In nine hypertensive patients, the distances between heart and renal artery level were almost identical due to their similar height, weight and ages. However, the optimal BSP TI was different for each subject, which hinted that the labeled blood's arriving time was different from heart to renal artery level due to various BFVs. For those subjects with BSP TI $=1400 \mathrm{~ms}$, their BVF should be the lowest, and vice versa. Therefore, the choice of optimal BSP TI could be determined by the patients' BFV in SLEEK for distinctly displaying the renal artery, and even evaluating renal artery diseases especially renal artery branch disease in the renal parenchyma. We recommend choosing optimal BSP TI based on BFV to avoid the repeat SLEEK scan with different BSP TIs and to improve work efficiency.

There were several limitations in our study. First, a $0.2 \mathrm{~cm}$ diameter catheter was used to simulate the renal artery, which is smaller and less stretched than the real renal artery. Second, the simulated device was not complex enough to represent the vessel in the individual's body. In conclusion, $\mathrm{BFV}$ has a negative relationship with BSP TI in SLEEK NCE-MRA. It is very vital to choose the optimal BSP TI based on BFV for improving the ability of delineating renal artery and to assess renal artery disease, which can enhance the diagnostic confidence and make a further treatment plan for patients.

\section{Footnotes}

Authors' Contributions: Study concept and design: Yigang Pei. Acquisition of data: Qian Ye, Juxiong Xiao. Analysis and interpretation of data: Xianjing Peng, Yigang Pei, Qian Ye, Juxiong Xiao. Drafting of the manuscript: Xianjing Peng, Yigang Pei. Critical revision of the manuscript for important intellectual content: Xianjing Peng, Yigang Pei. Statistical analysis: Yigang Pei. Administrative, technical, and material support: Hao Shen. Study supervision: Xueying Long.

Funding/Support: This work was supported by the National Natural Science Foundation of China (grant number: 81371541, Beijing, China), Natural Science Foundation of HuNan Province (grant number: 2018JJ2656, Changsha, China), Postdoctoral Science Foundation of Central South University.

Ethical Considerations: This study was approved by the institutional review board and written informed consent was obtained from all participants prior to examination.

\section{References}

1. Tang H, Wang Z, Wang L, Hu X, Wang Q, Li Z, et al. Depiction of transplant renal vascular anatomy and complications: Unenhanced MR angiography by using spatial labeling with multiple inversion pulses. 
Radiology. 2014;271(3):879-87. doi: 10.1148/radiol.14131800. [PubMed: 24592960].

2. Pei Y, Shen H, Li J, Zhang H, Xia L, Wang L, et al. Evaluation of renal artery in hypertensive patients by unenhanced MR angiography using spatial labeling with multiple inversion pulses sequence and by CT angiography. AJR Am J Roentgenol. 2012;199(5):1142-8. doi: 10.2214/AJR.11.7263. [PubMed: 23096191].

3. Yu PY, Chen MH, Ou HY, Huang TL, Yu CY, Chen CL, et al. Magnetic resonance angiographic inflow-sensitive inversion recovery technique for vascular evaluation before liver transplantation. Transplant Proc. 2014;46(3):682-5. doi: 10.1016/j.transproceed.2013.11.043. [PubMed: 24767323].

4. Xu JL, Shi DP, Li YL, Zhang JL, Zhu SC, Shen H. Non-enhanced MR angiography of renal artery using inflow-sensitive inversion recovery pulse sequence: A prospective comparison with enhanced CT angiography. Eur J Radiol. 2011;80(2):e57-63. doi: 10.1016/j.ejrad.2010.08.004. [PubMed: 20800405].

5. Albert TS, Akahane M, Parienty I, Yellin N, Catala V, Alomar X, et al. An international multicenter comparison of time-SLIP unenhanced MR angiography and contrast-enhanced CT angiography for assessing renal artery stenosis: The renal artery contrast-free trial. $A J R$ Am J Roentgenol. 2015;204(1):182-8. doi: 10.2214/AJR.13.12022. [PubMed: 25539255].

6. Hamamoto K, Matsuura K, Chiba E, Okochi T, Tanno K, Tanaka O. Feasibility of Non-contrast-enhanced MR angiography using the time-SLIP technique for the assessment of pulmonary arteriovenous malformation. Magn Reson Med Sci. 2016;15(3):253-65. doi: 10.2463/mrms.mp.2015-0069. [PubMed: 26841853]. [PubMed Central: PMC5608121].

7. Bultman EM, Klaers J, Johnson KM, Francois CJ, Schiebler ML, Reeder SB, et al. Non-contrast enhanced 3D SSFP MRA of the renal allograft vasculature: A comparison between radial linear combination and cartesian inflow-weighted acquisitions. Magn Reson Imaging. 2014;32(2):190-5. doi: 10.1016/j.mri.2013.10.004. [PubMed: 24246390]. [PubMed Central: PMC3893796].

8. Parienty I, Rostoker G, Jouniaux F, Piotin M, Admiraal-Behloul F, Miyazaki M. Renal artery stenosis evaluation in chronic kidney disease patients: Nonenhanced time-spatial labeling inversion-pulse three-dimensional MR angiography with regulated breathing versus DSA. Radiology. 2011;259(2):592-601. doi: 10.1148/radiol.11101422. [PubMed: 21330564]

9. Lanzman RS, Voiculescu A, Walther C, Ringelstein A, Bi X, Schmitt
P, et al. ECG-gated nonenhanced 3D steady-state free precession MR angiography in assessment of transplant renal arteries: Comparison with DSA. Radiology. 2009;252(3):914-21. doi: 10.1148/radiol.2531082260. [PubMed: 19635833].

10. Mohrs OK, Petersen SE, Schulze T, Zieschang M, Kux H, Schmitt P, et al. High-resolution 3D unenhanced ECG-gated respiratory-navigated MR angiography of the renal arteries: Comparison with contrastenhanced MR angiography. AJR Am J Roentgenol. 2010;195(6):1423-8. doi: 10.2214/AJR.10.4365. [PubMed: 21098205].

11. Shonai T, Takahashi T, Ikeguchi H, Miyazaki M, Amano K, Yui M. Improved arterial visibility using short-tau inversion-recovery (STIR) fat suppression in non-contrast-enhanced time-spatial labeling inversion pulse (Time-SLIP) renal MR angiography (MRA). J Magn Reson Imaging. 2009;29(6):1471-7. doi: 10.1002/jmri.21792. [PubMed: 19472424].

12. Kurata Y, Kido A, Fujimoto K, Kiguchi K, Takakura K, Moribata Y, et al. Optimization of non-contrast-enhanced MR angiography of the renal artery with three-dimensional balanced steady-state free-precession and time-spatial labeling inversion pulse (time-SLIP) at 3T MRI, in relation to age and blood velocity. Abdom Radiol (NY). 2016;41(1):119-26. doi: 10.1007/s00261-015-0608-4. [PubMed: 26830618].

13. Pei Y, Li F, Shen H, Long X, Liu H, Wang X, et al. Optimal blood suppression inversion time based on breathing rates and heart rates to improve renal artery visibility in spatial labeling with multiple inversion pulses: A preliminary study. Korean J Radiol. 2016;17(1):69-78. doi: 10.3348/kjr.2016.17.1.69. [PubMed: 26798218]. [PubMed Central: PMC4720814].

14. Tan ET, Huston J3, Campeau NG, Riederer SJ. Fast inversion recovery magnetic resonance angiography of the intracranial arteries. Magn Reson Med. 2010;63(6):1648-58. doi: 10.1002/mrm.22456. [PubMed: 20512868]. [PubMed Central: PMC2908948].

15. Kawel N, Jhooti P, Dashti D, Haas T, Winter L, Zellweger MJ, et al. MRimaging of the thoracic aorta: 3D-ECG- and respiratory-gated bSSFP imaging using the CLAWS algorithm versus contrast-enhanced 3DMRA. Eur J Radiol. 2012;81(2):239-43. doi: 10.1016/j.ejrad.2010.12.040. [PubMed: 21237595].

16. Shimizu H, Isoda H, Ohno T, Yamashita R, Kawahara S, Furuta A, et al. Non-contrast-enhanced MR portography and hepatic venography with time-spatial labeling inversion pulses: Comparison of imaging with the short tau inversion recovery method and the chemical shift selective method. Magn Reson Imaging. 2015;33(1):81-5. doi: 10.1016/j.mri.2014.08.013. [PubMed: 25159471]. 